ESAIM: COCV 20 (2014) 442-459

DOI: $10.1051 / \mathrm{cocv} / 2013070$
ESAIM: Control, Optimisation and Calculus of Variations

www.esaim-cocv.org

\title{
MINIMISING CONVEX COMBINATIONS OF LOW EIGENVALUES *
}

\author{
Mette IVersen $^{1}$ And DARIO MAZzoleni ${ }^{2,3}$
}

\begin{abstract}
We consider the variational problem

$$
\inf \left\{\alpha \lambda_{1}(\Omega)+\beta \lambda_{2}(\Omega)+(1-\alpha-\beta) \lambda_{3}(\Omega) \mid \Omega \text { open in } \mathbb{R}^{n},|\Omega| \leq 1\right\},
$$

for $\alpha, \beta \in[0,1], \alpha+\beta \leq 1$, where $\lambda_{k}(\Omega)$ is the $k$ th eigenvalue of the Dirichlet Laplacian acting in $L^{2}(\Omega)$ and $|\Omega|$ is the Lebesgue measure of $\Omega$. We investigate for which values of $\alpha, \beta$ every minimiser is connected.
\end{abstract}

Mathematics Subject Classification. 49Q10, 49R50, 35P15.

Received January 9, 2013. Revised June 28, 2013.

Published online March 7, 2014.

\section{INTRODUCTION}

Let $\Omega \subset \mathbb{R}^{n}$ be an open set with finite Lebesgue measure $|\Omega|$ and $\left\{\left(\lambda_{k}(\Omega), u_{k}(\Omega)\right)\right\}_{k \geq 1}$ denote respectively the eigenvalues and the eigenfunctions of the Dirichlet Laplacian acting in $L^{2}(\Omega)$ counted with their multiplicity, satisfying

$$
\left\{\begin{aligned}
-\Delta u_{k} & =\lambda_{k}(\Omega) u_{k} & & \text { in } \Omega \\
u_{k} & =0 & & \text { on } \partial \Omega .
\end{aligned}\right.
$$

The following min-max formula holds for eigenvalues ([12], Sect. 1.3),

$$
\lambda_{k}(\Omega)=\min _{\substack{E_{k} \subset H_{1}^{1}(\Omega) \\ k-\text { dimensional subspace }}} \max _{u \in E_{k}} \frac{\|D u\|_{L^{2}(\Omega)}^{2}}{\|u\|_{L^{2}(\Omega)}^{2}}, \quad k \in \mathbb{N},
$$

with the above ratio called the Rayleigh quotient. For general references about eigenvalues of Dirichlet Laplacian see for example $[11,12]$.

In this paper we are interested in the following variational problem:

$$
\inf \left\{\mathcal{F}(\Omega):=\alpha \lambda_{1}(\Omega)+\beta \lambda_{2}(\Omega)+(1-\alpha-\beta) \lambda_{3}(\Omega) \mid \Omega \text { open in } \mathbb{R}^{n},|\Omega| \leq 1\right\},
$$

Keywords and phrases. Eigenvalues, Dirichlet-Laplacian, Shape Optimization.

* This work has been supported by the ERC Starting Grant No. 258685 "AnOptSetCon".

1 School of Mathematics, University of Bristol, University Walk, Bristol BS8 1TW, United Kingdom. Mette.Iversen@bris.ac.uk

2 Dipartimento di Matematica, Università degli Studi di Pavia, Via Ferrata 1, 27100 Pavia, Italy. dario.mazzoleni@unipv.it

3 Department Mathematik, Friedrich-Alexander Universität Erlangen-Nürnberg, Cauerstrasse, 11, 91058 Erlangen, Germany. mazzoleni@math.fau.de 
for $\alpha, \beta \in[0,1]$ such that $\alpha+\beta \leq 1$. We recall that the following scaling properties hold for an open set $\Omega \subset \mathbb{R}^{n}$ :

$$
|t \Omega|=t^{n}|\Omega|, \quad \forall t>0 \quad \text { and } \quad \lambda_{k}(t \Omega)=t^{-2} \lambda_{k}(\Omega) \quad \text { for all } k \in \mathbb{N},
$$

hence it is clear that the unit measure in the constraint $|\Omega| \leq 1$ is for convenience only; everything works in the same way for any other positive constant. Moreover, thanks to (1.3), an optimal set must satisfy the equality in the measure constraint. The existence of a quasi-open minimiser for (1.2) follows from Theorem A of the recent paper [13].

The aim of this paper is to show that any minimiser for (1.2) is connected for a range of values of $\alpha, \beta$, and to discuss the remaining cases.

When $\alpha=1$ and $\beta=0$, problem (1.2) reduces to the minimisation of the first eigenvalue, which is a very interesting topic studied first by Lord Rayleigh in the 19th century. Faber and Krahn proved (see [12], Thm. 3.2.1) that:

$$
\lambda_{1}(\Omega) \geq \lambda_{1}(B)\left(\frac{|B|}{|\Omega|}\right)^{2 / n}
$$

where $B$ is the ball of unit measure in $\mathbb{R}^{n}$, and with equality if and only if $\Omega$ is any ball (up to sets of capacity zero). Hence in this case there is an unique minimiser - the ball - which is connected in every dimension. On the other hand, if we consider the case of $\alpha=0$ and $\beta=1$, which means that we are minimising the second eigenvalue, the Krahn-Szegö inequality (see [12], Thm. 4.1.1) asserts that

$$
\lambda_{2}(\Omega) \geq 2^{2 / n} \lambda_{1}(B)\left(\frac{|B|}{|\Omega|}\right)^{2 / n},
$$

with equality if and only if $\Omega$ is any disjoint union of two balls of equal measure. Thus in this case the minimiser -two disjoint equal balls- is disconnected in each dimension. We will denote the union of two disjoint balls each of half measure by $\Theta$.

Very little is known about the other cases: Wolf and Keller in [16] proved that in dimension $n=2,3$ any minimiser for $\lambda_{3}(\Omega)$ (which corresponds to $\alpha=\beta=0$ in (1.2)) is connected, by showing that the ball has lower third eigenvalue than any disconnected set of the same measure. Moreover they conjectured the ball to be the minimiser for $\lambda_{3}(\Omega)$ in $\mathbb{R}^{2}$. Connectedness of minimisers for individual eigenvalues is studied in [5].

Before stating the main results, we define some constants which will be useful in the following.

Throughout this paper let $\alpha_{n}$ satisfy

$$
\alpha_{n}=\frac{\lambda_{2}(B)-2^{2 / n} \lambda_{1}(B)}{\lambda_{2}(B)-\lambda_{1}(B)}
$$

for $n=2,3,4$, and be the infimum of the numbers that satisfies

$$
\alpha\left[\left(\left(\frac{1-\alpha}{\alpha}\right)^{n /(n+2)}+1\right)^{2 / n}-1\right]+(1-\alpha)\left[\left(\left(\frac{\alpha}{1-\alpha}\right)^{n /(n+2)}+1\right)^{2 / n}-\frac{\lambda_{2}(B)}{\lambda_{1}(B)}\right]>0,
$$

for $n \geq 5$. Let $\beta_{2}=2-\frac{\lambda_{2}(B)}{2 \lambda_{1}(B)}, \beta_{3}$ be the supremum of the numbers in the range $\left(\frac{1}{3}, \frac{2^{2 / 3}}{1+2^{2 / 3}}\right)$, satisfying

$$
\beta\left[2^{2 / 5}\left(\frac{1-\beta}{\beta}\right)^{3 / 5}+1\right]^{2 / 3}+2^{2 / 3}(1-\beta)\left[2^{-2 / 5}\left(\frac{\beta}{1-\beta}\right)^{3 / 5}+1\right]^{2 / 3}-\frac{\lambda_{2}(B)}{\lambda_{1}(B)}>0,
$$

and let $\beta_{n}=0$ for $n \geq 4$. Finally let $\gamma_{2}=\gamma_{3}=0$ and let $\gamma_{n}$ for $n \geq 4$ be the infimum of the numbers satisfying

$$
\gamma\left[\left(1+\left(\frac{\lambda_{1}(B)}{\lambda_{2}(B)}\right)^{n / 2}\right)^{2 / n}-1\right]+(1-\gamma)\left[3^{2 / n}-\frac{\lambda_{2}(B)}{\lambda_{1}(B)}\right]>0 .
$$


The appoximate values for $n=2,3,4$ are:

\begin{tabular}{cccc}
\hline$n$ & $\alpha_{n}$ & $\beta_{n}$ & $\gamma_{n}$ \\
\hline 2 & 0.350 & 0.730 & 0 \\
3 & 0.439 & 0.476 & 0 \\
4 & 0.479 & 0 & 0.311 \\
\hline
\end{tabular}

We are now in position to state the main theorems, the first one in general dimension, with the second giving additional information about the two dimensional case.

Theorem 1.1. Any minimiser of (1.2) is connected for each of the cases

(i) $\alpha+\beta=1, \alpha>0$;

(ii) $\alpha_{n}<\alpha \leq 1$;

(iii) $0<\beta<\beta_{n}(1-\alpha)$;

(iv) $\beta=0, \gamma_{n}<\alpha \leq 1$.

Remark 1.2. For $n=2$, Theorem 1.1 states that every minimiser for (1.2) is connected in each of the cases:

(i) $\alpha+\beta=1, \alpha>0$

(ii) $0.350 \approx \alpha_{2}<\alpha \leq 1$;

(iii) $0 \leq \beta<\beta_{2}(1-\alpha) \approx 0.730(1-\alpha)$,

Theorem 1.3. Let $n=2$.

(a) Any disconnected minimiser of (1.2) satisfies $\lambda_{1}(\Omega)=\lambda_{2}(\Omega)$ and has exactly two components.

(b) If any minimiser of (1.2) is connected for $\alpha=0$ and each $\beta \in[0,1)$, then any minimiser is connected unless $\beta=1$.

From Remark 1.2 and Theorem 1.3 it is quite natural to make the following conjecture.

Conjecture 1.4. Let $n=2$; a minimiser for the problem (1.2) can not be disconnected unless $\beta=1$.

In a recent paper by Osting and Kao [14], there are numerical results that support Conjecture 1.4: the numerically computed optimal domain for problem (1.2) has one connected component unless $\alpha=0$ and $\beta=1$. Moreover the numerical computations also suggest that in the region $\{(\alpha, \beta): \alpha+\beta \leq 1\}$ the optimal solution is a ball, while for all the other $(\alpha, \beta)$-values (except of course $(\alpha, \beta)=(0,1)$ ) the first four eigenvalues of the optimal domain are each simple. It is interesting to note that this last numerical result, together with Theorem 1.3 (a) supports Conjecture 1.4, too.

This paper is organised as follows. In Section 2 we recall the values for the eigenvalues of the ball and prove that a disjoint union of two balls can not be optimal for (1.2) unless $\beta=1$. Section 3 contains the proof of Theorem 1.1, while in Section 4 we focus on the two dimensional case: after showing that a disconnected minimiser has at most two connected components and must have multiple eigenvalues, we prove Theorem 1.3.

\section{Preliminaries}

First of all we recall the value of the eigenvalues for the unit ball $B$, which we will use many times for calculations: $\lambda_{1}(B)=\omega_{n}^{2 / n} j_{n / 2-1}^{2}$, and $\lambda_{2}(B)=\ldots=\lambda_{n+1}(B)=\omega_{n}^{2 / n} j_{n / 2}^{2}$, where $\omega_{n}$ denotes the volume of the ball of unit radius in $\mathbb{R}^{n}$, and $j_{\nu}$ is the first positive zero of the Bessel function $J_{\nu}$. The approximate values of the zeros of Bessel functions can be found in [1]; we will always consider only three decimal digits. In $\mathbb{R}^{2}$ we have $\lambda_{1}(B) \approx 18.168$ and $\lambda_{2}(B)=\lambda_{3}(B) \approx 46.125$. Throughout the paper we will for convenience define the value $m_{0}=\frac{\lambda_{1}(B)}{\lambda_{2}(B)}$, while the values of $m_{1}, m_{2}$ will denote, respectively, the lower and the upper bound for the measure of a connected component. We will use in Section 4 a couple of fundamental results obtained by Ashbaugh and Benguria in $[2,3]$; we restate them here. 
Theorem 2.1. The ball maximises the ratio $\lambda_{2} / \lambda_{1}$, that is:

$$
\frac{\lambda_{2}(\Omega)}{\lambda_{1}(\Omega)} \leq \frac{\lambda_{2}(B)}{\lambda_{1}(B)} \quad \text { for all open sets } \Omega \subset \mathbb{R}^{n} .
$$

Moreover two equal disjoint balls maximise the ratio $\lambda_{3} / \lambda_{2}$, hence

$$
\frac{\lambda_{3}(\Omega)}{\lambda_{2}(\Omega)} \leq \frac{\lambda_{3}(\Theta)}{\lambda_{2}(\Theta)}=\frac{\lambda_{2}(B)}{\lambda_{1}(B)} \quad \text { for all open sets } \Omega \subset \mathbb{R}^{n} .
$$

In Sections 3 and $4 \Omega$ it is used to denote an optimal disconnected candidate for a minimiser. Note that for disconnected sets the eigenvalues are obtained by collecting and reordering the eigenvalues of the components (see [12], Rem. 1.2.4). In view of that we give the following definition.

Definition 2.2. We say that the $k$ th eigenfunction $u_{k}$ is supported on a component $G$ of $\Omega$ when $\lambda_{k}(\Omega)=\lambda_{i}(G)$, for some $i \leq k$. Moreover we write that $G$ supports $l$ eigenvalues if it has $l$ eigenvalues less than or equal to the largest eigenvalue of $\Omega$ that we are minimising.

Note that a minimiser for (1.2) must have at least one of the first three eigenfunctions supported on each component.

An important component in the proof of Theorem 1.1 is the following lemma.

Lemma 2.3. Let $n \geq 2$. The disjoint union of two balls can be optimal for (1.2) only if $\beta=1$.

Proof. The idea of the proof is that letting the two disjoint equal balls slightly overlap we obtain a better candidate for a minimiser of (1.2), because the increase in the second eigenvalue is less than the decrease in the first and the third. We divide the proof in two steps, treating first the case of two balls with equal measure, then the case of balls with different size.

Step I. Let $B(\varepsilon)=B(0,1) \cap\left\{x \mid x_{1}<1-\varepsilon\right\}$ and $\Omega(\varepsilon)=B(0,1) \cup B\left(2(1-\varepsilon) e_{1}, 1\right)$, where $x=\left(x_{1}, x_{2}, \ldots, x_{n}\right)$ and $e_{1}$ is the unit vector in the $x_{1}$ direction. Moreover $\tilde{\Omega}(\varepsilon)=|\Omega(\varepsilon)|^{-\frac{1}{n}} \Omega(\varepsilon)$ is the set rescaled to unit measure. It follows from Theorem 1 in [4] that

$$
\lambda_{1}(B(\varepsilon))|B(\varepsilon)|^{2 / n}=\lambda_{1}(B)|B|^{2 / n}+o\left(\varepsilon^{(n+1) / 2}\right) .
$$

Since eigenvalues of Dirichlet Laplacian are monotone with respect to set inclusion, we have $\lambda_{1}(\Omega(\varepsilon))<\lambda_{1}(B)$ and $\lambda_{3}(\Omega(\varepsilon))<\lambda_{2}(B)$. Thus taking scaling into account gives $\lambda_{1}(\tilde{\Omega}(\varepsilon))<\lambda_{1}(\Theta)-c_{1} \varepsilon^{(n+1) / 2}$ and $\lambda_{3}(\tilde{\Omega}(\varepsilon))<$ $\lambda_{3}(\Theta)-c_{2} \varepsilon^{(n+1) / 2}$, for some positive constants $c_{1}, c_{2}$, reminding that $\lambda_{2}(B)=\lambda_{3}(B), \lambda_{1}(\Theta)=2^{2 / n} \lambda_{1}(B)$ and $\lambda_{3}(\Theta)=2^{2 / n} \lambda_{2}(B)$. By the min-max principle (1.1) we can obtain an upper bound for $\lambda_{2}(\Omega(\varepsilon))$ by choosing the subspace $E_{2} \subset H_{0}^{1}(\Omega)$ spanned by the first eigenfunction of $B(\varepsilon)$ and the first eigenfunction of $\Omega(\varepsilon) \cap\left\{x \mid x_{1}>1-\varepsilon\right\}$. Hence $\lambda_{2}(\Omega(\varepsilon)) \leq \lambda_{1}(B(\varepsilon)$ ), so we can apply (2.3) and use the scaling (1.3) to obtain $\lambda_{2}(\tilde{\Omega}(\varepsilon)) \leq \lambda_{2}(\Theta)+o\left(\varepsilon^{(n+1) / 2}\right)$.

For $\beta<1$ and for sufficiently small $\varepsilon>0$, this gives

$$
\begin{aligned}
\alpha \lambda_{1}(\tilde{\Omega}(\varepsilon)) & +\beta \lambda_{2}(\tilde{\Omega}(\varepsilon))+(1-\alpha-\beta) \lambda_{3}(\tilde{\Omega}(\varepsilon)) \\
& <\alpha \lambda_{1}(\Theta)+\beta \lambda_{2}(\Theta)+(1-\alpha-\beta) \lambda_{3}(\Theta) .
\end{aligned}
$$

Step II. Let $r_{1}>r_{2}$ and $\Omega$ be the disjoint union of two balls with radii $r_{1}, r_{2}$ such that the first two eigenfunctions are supported on different components. We write $\tilde{\Omega}=|\Omega|^{-\frac{1}{n}} \Omega$ for the set rescaled to unit measure. Then we define

$$
\begin{array}{r}
B_{r_{1}}=B\left(0, r_{1}\right), \quad B_{r_{2}}=B\left(\left(r_{1}+r_{2}-\frac{\varepsilon}{2}\left(\frac{1}{r_{1}}+\frac{1}{r_{2}}\right)\right) e_{1}, r_{2}\right), \quad \Omega(\varepsilon)=B_{r_{1}} \cup B_{r_{2}}, \\
B_{1}(\varepsilon)=B_{r_{1}} \cap\left\{x \mid x_{1}<r_{1}-\frac{\varepsilon}{2 r_{1}}\right\}, \quad B_{2}(\varepsilon)=B_{r_{2}} \cap\left\{x \mid x_{1}>r_{2}-\frac{\varepsilon}{2 r_{2}}\right\} .
\end{array}
$$

Figure 1 represents a possible configuration of the sets above. 


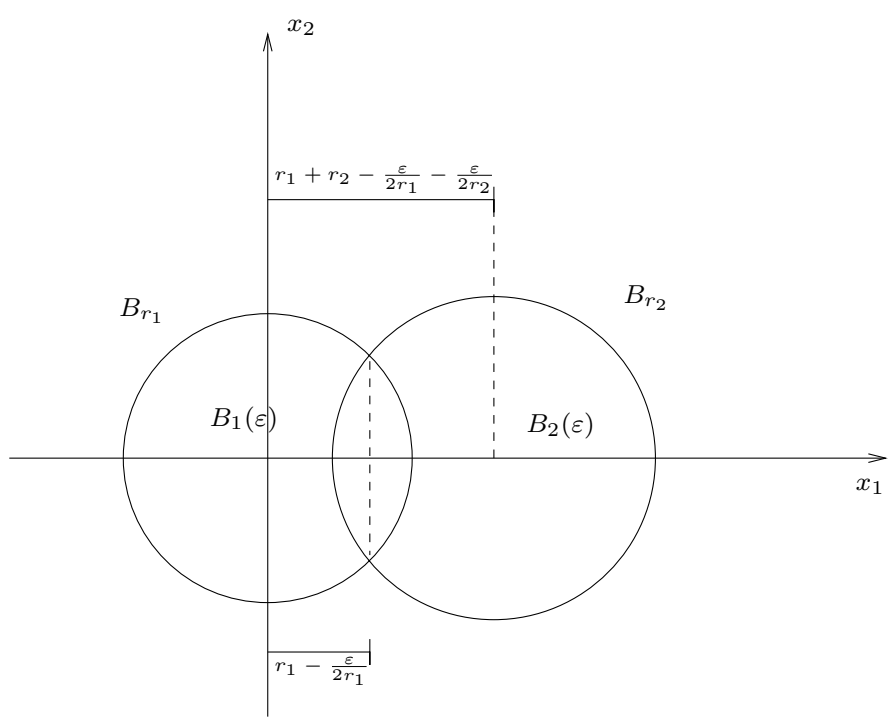

Figure 1. The sets $B_{r_{1}}, B_{r_{2}}, B_{1}(\varepsilon)$ and $B_{2}(\varepsilon)$.

By monotonicity of Dirichlet eigenvalues with respect to set inclusion, we have $\lambda_{1}(\Omega(\varepsilon))<\lambda_{1}\left(B_{r_{1}}\right)$ and $\lambda_{3}(\Omega(\varepsilon))<\lambda_{2}\left(B_{r_{1}}\right)$, and again taking account of the scaling we have $\lambda_{1}(\tilde{\Omega}(\varepsilon))<\lambda_{1}(\tilde{\Omega})-c_{1} \varepsilon^{(n+1) / 2}$ and $\lambda_{3}(\tilde{\Omega}(\varepsilon))<\lambda_{3}(\tilde{\Omega})-c_{2} \varepsilon^{(n+1) / 2}$, for some positive constants $c_{1}, c_{2}$.

By the min-max principle (1.1), we can obtain an upper bound for $\lambda_{2}(\Omega(\varepsilon))$ by choosing $E_{2} \subset H_{0}^{1}(\Omega)$ spanned by the first eigenfunction of $B_{r_{2}}$ and the first eigenfunction of $\Omega(\varepsilon) \backslash B_{r_{2}}$, and for $\varepsilon$ small enough we have $\lambda_{1}\left(B_{r_{2}}\right) \leq \lambda_{1}\left(\Omega(\varepsilon) \backslash B_{r_{2}}\right)$. Hence $\lambda_{2}(\Omega(\varepsilon)) \leq \lambda_{1}\left(B_{r_{2}}\right)$, and taking account of the scaling $\lambda_{2}(\tilde{\Omega}(\varepsilon)) \leq$ $\lambda_{2}(\tilde{\Omega})-c_{3} \varepsilon^{(n+1) / 2}$, for some positive $c_{3}$. In conclusion, for $\beta<1$ and $\varepsilon$ small enough, $\tilde{\Omega}(\varepsilon)$ is a better candidate than $\tilde{\Omega}$ for problem (1.2).

This last remark will be useful in Sections 3 and 4 .

Remark 2.4. Let $n=2,3$. A disconnected set $\Omega$ can never be optimal for (1.2) if $\lambda_{2}(\Omega) \geq \lambda_{2}(B)$. Here the ball is better, since $\lambda_{1}(B)<\lambda_{1}(\Omega)$ by the Faber-Krahn inequality and $\lambda_{3}(B)<\lambda_{3}(\Omega)$ by ([12], Cor. 5.2.2).

\section{THE GENERAL CASE}

This section is completely devoted to the proof of Theorem 1.1. Before starting the proof we note that if a connected component of the optimal disconnected set supports only one of the first three eigenfunctions, then by the Faber-Krahn inequality it must be a ball of the same measure. We remind that we call $\left(u_{i}\right)_{i=1,2,3}$ the eigenfunctions related to the eigenvalues $\left(\lambda_{i}\right)_{i=1,2,3}$.

Proof of Theorem 1.1 (i). We deal with the case $\alpha+\beta=1$, that is, we consider the functional $\alpha \lambda_{1}(\cdot)+(1-$ $\alpha) \lambda_{2}(\cdot)$. Note that this result for $\mathbb{R}^{2}$ is also discussed in ([15], Chap. 2), but the details of a proof are not given. A disconnected minimiser $\Omega$ must by the Faber-Krahn inequality be the union of two disjoint balls, since we are considering only the first two eigenvalues. Hence an immediate application of Lemma 2.3 rules out this configuration in any dimension when $\alpha>0$, so we conclude.

Proof of Theorem 1.1 (ii). We need a different argument from the one above, but start again from the case $\alpha+\beta=1$. The case $\alpha=1$ was already solved by the Faber-Krahn inequality (1.4). Again a disconnected minimiser $\Omega$ must be the union of two disjoint balls. Without loss of generality, we suppose that the ball 
supporting $u_{1}$ has measure $m \leq 1$, while the other one (supporting $u_{2}$ ) has measure $1-m$. Having in mind the scaling of eigenvalues, it is clear that $m \geq 1 / 2$ and we have

$$
\alpha \lambda_{1}(\Omega)+(1-\alpha) \lambda_{2}(\Omega)=\lambda_{1}(B)\left(\frac{\alpha}{m^{2 / n}}+\frac{1-\alpha}{(1-m)^{2 / n}}\right) .
$$

First of all we consider the case when $\alpha \in\left(\frac{1}{2}, 1\right)$. By minimising in $m$ in the previous expression to obtain a lower bound and comparing with the value for the unit ball rules out this configuration if

$$
\alpha \lambda_{1}(B)\left[\left(\frac{1-\alpha}{\alpha}\right)^{n /(n+2)}+1\right]^{2 / n}+(1-\alpha) \lambda_{1}(B)\left[\left(\frac{\alpha}{1-\alpha}\right)^{n /(n+2)}+1\right]^{2 / n}
$$

that is, when the function $f_{n}:(0,1) \rightarrow \mathbb{R}$,

$$
f_{n}(\alpha)=\alpha\left\{\left[\left(\frac{1-\alpha}{\alpha}\right)^{\frac{n}{n+2}}+1\right]^{\frac{2}{n}}-1\right\}+(1-\alpha)\left\{\left[\left(\frac{\alpha}{1-\alpha}\right)^{\frac{n}{n+2}}+1\right]^{\frac{2}{n}}-\frac{\lambda_{2}(B)}{\lambda_{1}(B)}\right\}
$$

is positive. The following property of $f_{n}(\alpha)$ is important for our analysis.

Claim A. For every $n \in \mathbb{N}$, there exists $\alpha_{n} \in(0,1)$ such that

$$
f_{n}(\alpha)<0 \quad \text { if } \alpha \in\left(0, \alpha_{n}\right) \quad \text { and } \quad f_{n}(\alpha)>0 \quad \text { if } \alpha \in\left(\alpha_{n}, 1\right) .
$$

Proof of Claim A. We introduce the increasing function $\psi:[0,1) \rightarrow[0, \infty)$ :

$$
\psi(\alpha)=\frac{\alpha}{1-\alpha},
$$

and $\phi_{n}:(0, \infty) \rightarrow \mathbb{R}$

$$
\phi_{n}(t)=t\left\{\left[\left(\frac{1}{t}\right)^{\frac{n}{n+2}}+1\right]^{\frac{2}{n}}-1\right\}+\left\{\left[t^{\frac{n}{n+2}}+1\right]^{\frac{2}{n}}-\frac{\lambda_{2}(B)}{\lambda_{1}(B)}\right\},
$$

so that $f_{n}(\alpha)=(1-\alpha) \phi_{n}(\psi(\alpha))$, for $\alpha \in(0,1)$. Hence the sign of $f_{n}$ in the interval $(0,1)$ is the same as that of $\phi_{n}$ in the interval $(0, \infty)$. We note that $\phi_{n}$ is the sum of two functions, where the second one,

$$
t \mapsto\left(t^{\frac{n}{n+2}}+1\right)^{\frac{2}{n}}-\frac{\lambda_{2}(B)}{\lambda_{1}(B)}
$$

is strictly increasing. The first one,

$$
t \mapsto t\left\{\left[\left(\frac{1}{t}\right)^{\frac{n}{n+2}}+1\right]^{\frac{2}{n}}-1\right\}=\phi_{n}^{1}(t),
$$

is also strictly increasing, since we have:

$$
\frac{\mathrm{d}}{\mathrm{d} t} \phi_{n}^{1}(t)=\left\{\left[\left(\frac{1}{t}\right)^{\frac{n}{n+2}}+1\right]^{\frac{2}{n}}-1\right\}-\frac{2}{n+2} t^{-\frac{n}{n+2}}\left[\left(\frac{1}{t}\right)^{\frac{n}{n+2}}+1\right]^{\frac{2}{n}-1}
$$


that is positive when

$$
1+\frac{n}{n+2} t^{-\frac{n}{n+2}}>\left[\left(\frac{1}{t}\right)^{\frac{n}{n+2}}+1\right]^{1-\frac{2}{n}} .
$$

The above inequality holds, since $t \mapsto t^{1-2 / n}$ is concave and thus

$$
\left[\left(\frac{1}{t}\right)^{\frac{n}{n+2}}+1\right]^{1-\frac{2}{n}}<1+\frac{n-2}{n}\left(\frac{1}{t}\right)^{\frac{n}{n+2}}<1+\frac{n}{n+2}\left(\frac{1}{t}\right)^{\frac{n}{n+2}},
$$

where we used also the inequality $(n-2) / n<n /(n+2)$. So $\phi_{n}$ is the sum of two strictly increasing functions and thus it has the same property. Hence $\phi_{n}$ can change sign only once in $(0, \infty)$. We note that

$$
\lim _{t \rightarrow 0^{+}} \phi_{n}(t)=1-\frac{\lambda_{2}(B)}{\lambda_{1}(B)}<0 \text { and } \lim _{t \rightarrow \infty} \phi_{n}(t)=+\infty
$$

so there exists an unique $t_{n} \in(0, \infty)$ such that

$$
\phi_{n}(t)<0 \text { if } t \in\left(0, t_{n}\right) \text { and } \phi_{n}(t)>0 \text { if } t \in\left(t_{n}, \infty\right) .
$$

In conclusion the thesis follows setting $\alpha_{n}=\psi^{-1}\left(t_{n}\right)$.

Keep in mind that, for all $n \geq 2, f_{n}(\alpha)>0$ if and only if (3.1) holds for $\alpha$. We can compute

$$
\begin{aligned}
& f_{2}\left(\frac{1}{2}\right)=\frac{1}{2}((1+1)-1)+\frac{1}{2}\left[(1+1)-\frac{\lambda_{2}(B)}{\lambda_{1}(B)}\right] \approx 0.230>0, \\
& f_{3}\left(\frac{1}{2}\right)=\frac{1}{2}\left((1+1)^{2 / 3}-1\right)+\frac{1}{2}\left[(1+1)^{2 / 3}-\frac{\lambda_{2}(B)}{\lambda_{1}(B)}\right] \approx 0.065>0, \\
& f_{4}\left(\frac{1}{2}\right)=\frac{1}{2}\left((1+1)^{1 / 2}-1\right)+\frac{1}{2}\left[(1+1)^{1 / 2}-\frac{\lambda_{2}(B)}{\lambda_{1}(B)}\right] \approx 0.016>0,
\end{aligned}
$$

where we used that

$$
\frac{\lambda_{2}(B)}{\lambda_{1}(B)} \approx \begin{cases}2.539 & \text { if } n=2 \\ 2.044 & \text { if } n=3 \\ 1.796 & \text { if } n=4\end{cases}
$$

Then, by Claim A, for $n \leq 4$ we have that $\alpha_{n}<1 / 2$, since $f_{n}(1 / 2)>0$. Hence $f_{n}(\alpha)>0$ for all $\alpha \in(1 / 2,1)$ and thus (3.1) holds in that range.

When $n \geq 5$, we define $\widetilde{\alpha}_{n}=\inf \{\alpha \in(1 / 2,1) \mid(3.1)$ holds $\}$ and Claim A assures that (3.1) is true for all $\alpha \in\left(\widetilde{\alpha}_{n}, 1\right)$.

Now we deal with the case when $\alpha \in\left(0, \frac{1}{2}\right]$. The constraint $m \geq 1 / 2$ implies that the optimal disconnected configuration consists in two disjoint balls of equal measure, since in this case we have

$$
\lambda_{1}(B) 2^{2 / n}=\alpha \lambda_{1}(\Theta)+(1-\alpha) \lambda_{2}(\Theta)<\lambda_{1}(B)\left(\frac{\alpha}{m^{2 / n}}+\frac{1-\alpha}{(1-m)^{2 / n}}\right) .
$$

This is ruled out by comparison with the unit ball when

$$
\lambda_{1}(B) 2^{2 / n}>\alpha \lambda_{1}(B)+(1-\alpha) \lambda_{2}(B),
$$

and we call, for $n \leq 4, \widehat{\alpha}_{n}=\frac{\lambda_{2}(B)-2^{\frac{2}{n}} \lambda_{1}(B)}{\lambda_{2}(B)-\lambda_{1}(B)}$, so that if $\alpha \in\left(\widehat{\alpha}_{n}, 1 / 2\right]$, then (3.3) holds. On the other hand, if $n \geq 5,(3.3)$ is never true. 
In conclusion, putting together the discussion for $\alpha \in(0,1 / 2]$ and for $\alpha \in(1 / 2,1)$, we have that

(a) For $n \leq 4$ the ball is a better candidate for problem (1.2) than any disconnected minimiser if $\alpha \in\left(\widehat{\alpha}_{n}, 1 / 2\right] \cup$ $(1 / 2,1)$.

(b) For $n \geq 5$ the ball is a better candidate for problem (1.2) than any disconnected minimiser if $\alpha \in\left(\widetilde{\alpha}_{n}, 1\right)$.

Thus we define $\alpha_{n}=\widetilde{\alpha}_{n}$ if $n \geq 5$ and $\alpha_{n}=\widehat{\alpha}_{n}$ if $n \leq 4$; hence we have that the ball is better than any disconnected set for $1 \geq \alpha>\alpha_{n}$.

Finally to extend beyond the situation $\alpha+\beta=1$, just note that for $1 \geq \alpha>\alpha_{n}$

$$
\begin{aligned}
\alpha \lambda_{1}(\Omega) & +\beta \lambda_{2}(\Omega)+(1-\alpha-\beta) \lambda_{3}(\Omega) \geq \alpha \lambda_{1}(\Omega)+(1-\alpha) \lambda_{2}(\Omega) \\
& >\alpha \lambda_{1}(B)+(1-\alpha) \lambda_{2}(B)=\alpha \lambda_{1}(B)+\beta \lambda_{2}(B)+(1-\alpha-\beta) \lambda_{3}(B),
\end{aligned}
$$

using the fact that $\lambda_{2}(\Omega) \leq \lambda_{3}(\Omega)$ while $\lambda_{2}(B)=\lambda_{3}(B)$, so we conclude.

Proof of Theorem 1.1 (iii). The case $\alpha=0$. We first consider the case $\alpha=0$, that is, we deal with the functional $\beta \lambda_{2}(\cdot)+(1-\beta) \lambda_{3}(\cdot)$. First of all we find out the best disconnected configuration.

Claim B. Let $\alpha=0$. A disconnected minimiser is made by a ball supporting $u_{2}$ and another set supporting $u_{1}$ and $u_{3}$.

Proof of Claim B. At first, we consider the configuration made by a set supporting $u_{1}, u_{2}$ and a ball supporting $u_{3}$. Since $\lambda_{1}$ does not appear in the functional, it is better to have three balls by applying Krahn-Szegö inequality (1.5) to the set supporting $u_{1}$ and $u_{2}$. Note that the new set, made by three disjoint balls, is as in the statement of the claim.

On the other hand an optimal configuration made by a ball supporting $u_{1}$ and by another set supporting $u_{2}$, $u_{3}$ should satisfy $\lambda_{1}(\Omega)=\lambda_{2}(\Omega)$, since $\lambda_{1}$ is not involved in the minimisation. Up to switch $\lambda_{1}$ and $\lambda_{2}$, we are in a configuration made by a ball supporting $u_{2}$ and another set supporting $u_{1}, u_{3}$ so the claim is proved.

Thanks to Claim B, it remains only to rule out a disconnected minimiser made by a ball supporting $u_{2}$, which we suppose to have measure $m$ (hence $\lambda_{2}(\Omega)=\frac{\lambda_{1}(B)}{m^{2 / n}}$ ) and a set supporting $u_{1}$ and $u_{3}$ (which must have measure $1-m)$. Unfortunately, we are able to find out informations only when $n=2,3$. First of all we note that $m \leq 1 / 2$, otherwise, by the Faber-Krahn inequality, $\lambda_{1}(\Omega)>\lambda_{2}(\Omega)$. Moreover, using Remark 2.4, it must happen that $\lambda_{2}(B)>\lambda_{2}(\Omega)$, which implies

$$
\frac{\lambda_{1}(B)}{m^{2 / n}}<\lambda_{2}(B), \quad \text { that is, } \quad m>\left(\frac{\lambda_{1}(B)}{\lambda_{2}(B)}\right)^{n / 2},
$$

that assures $m \geq 1 / 3$ for $n=2,3$. For $\beta \in[0,1)$, the Krahn-Szegö inequality gives the lower bound

$$
\beta \lambda_{2}(\Omega)+(1-\beta) \lambda_{3}(\Omega) \geq \lambda_{1}(B)\left(\frac{\beta}{m^{2 / n}}+\frac{2^{2 / n}(1-\beta)}{(1-m)^{2 / n}}\right) .
$$

We are interested in minimising the left hand side of (3.5) with respect to $m$, in order to improve the lower bound. We define

$$
m(\beta)=\left[2^{2 /(n+2)}\left(\frac{1-\beta}{\beta}\right)^{n /(n+2)}+1\right]^{-1},
$$

and note that the right hand side of (3.5) is decreasing in $(0, m(\beta))$ and increasing in $(m(\beta), \infty)$. Moreover, we have that

$$
m(\beta) \leq \frac{1}{3} \quad \text { if } \beta \in\left[0, \frac{1}{3}\right] \quad \text { and } \quad m(\beta) \geq \frac{1}{2} \quad \text { if } \beta \in\left[\frac{2^{\frac{2}{n}}}{1+2^{\frac{2}{n}}}, 1\right),
$$


so that (from the constraints on $m$ ) in this two ranges the right hand side of (3.5) is minimal for $m=1 / 3$ and $m=1 / 2$ respectively. On the other hand, when $\beta \in\left(\frac{1}{3}, \frac{2^{\frac{2}{n}}}{1+2^{\frac{2}{n}}}\right)$, the right hand side of $(3.5)$ is minimal for $m=m(\beta)$.

We are now in position to compare the lower bound for $\beta \lambda_{2}(\Omega)+(1-\beta) \lambda_{3}(\Omega)$ with $\beta \lambda_{2}(B)+(1-\beta) \lambda_{3}(B)=$ $\lambda_{2}(B)$.

For $\beta \in[0,1 / 3]$ we have

$$
\lambda_{2}(B)<3^{\frac{2}{n}} \lambda_{1}(B) \leq \beta \lambda_{2}(\Omega)+(1-\beta) \lambda_{3}(\Omega),
$$

hence in this range, for $n=2,3$, the ball is a better candidate than any disconnected set for problem (1.2).

Now we deal with the case when $\beta \in\left(\frac{1}{3}, \frac{2^{2 / n}}{1+2^{2 / n}}\right)$. Substituting the optimal values for $m$ in $(3.5)$ and comparing with $\beta \lambda_{2}(B)+(1-\beta) \lambda_{3}(B)=\lambda_{2}(B)$ gives connectedness for the whole range when $n=2$, since

$$
\lambda_{2}(B)<\lambda_{1}(B)\left[\beta\left(\sqrt{\frac{2(1-\beta)}{\beta}}+1\right)+2(1-\beta)\left(\sqrt{\frac{\beta}{2(1-\beta)}}+1\right)\right] \quad \forall \beta \in\left(\frac{1}{3}, \frac{2}{3}\right) .
$$

On the other hand, for $n=3$, we have connectedness when

$$
\lambda_{2}(B)<\lambda_{1}(B)\left\{\beta\left[2^{2 / 5}\left(\frac{1-\beta}{\beta}\right)^{3 / 5}+1\right]^{2 / 3}+2^{2 / 3}(1-\beta)\left[2^{-2 / 5}\left(\frac{\beta}{1-\beta}\right)^{3 / 5}+1\right]^{2 / 3}\right\} .
$$

In order to study this situation, we start by considering the function $g:(0,1) \rightarrow \mathbb{R}$

$$
g(\beta)=\beta\left[2^{2 / 5}\left(\frac{1-\beta}{\beta}\right)^{3 / 5}+1\right]^{2 / 3}+2^{2 / 3}(1-\beta)\left[2^{-2 / 5}\left(\frac{\beta}{1-\beta}\right)^{3 / 5}+1\right]^{2 / 3}-\frac{\lambda_{2}(B)}{\lambda_{1}(B)},
$$

and note that (3.6) holds for a $\beta \in\left(\frac{1}{3}, \frac{2^{2 / 3}}{1+2^{2 / 3}}\right)$ if and only if $g(\beta)>0$. The function $g$ is concave in its whole domain $(0,1)$, since it is possible to compute

$$
\begin{aligned}
g^{\prime \prime}(\beta)= & -\left(\frac{2^{9 / 5}}{25} \beta^{-11 / 5}(1-\beta)^{-2 / 5}\right)\left[2^{2 / 5}\left(\frac{1-\beta}{\beta}\right)^{3 / 5}+1\right]^{-4 / 3} \\
& -\frac{2^{9 / 5}}{25}\left(\beta^{-3 / 5}(1-\beta)^{-7 / 5}+\beta^{-8 / 5}(1-\beta)^{-2 / 5}\right)\left[2^{2 / 5}\left(\frac{1-\beta}{\beta}\right)^{3 / 5}+1\right]^{-1 / 3} \\
& -\frac{2^{13 / 15}}{25} \beta^{-4 / 5}(1-\beta)^{-11 / 5}\left[2^{-2 / 5}\left(\frac{\beta}{1-\beta}\right)^{3 / 5}+1\right]^{-4 / 3} \\
& -\frac{2^{34 / 15}}{25}\left(\beta^{-2 / 5}(1-\beta)^{-8 / 5}+\beta^{-7 / 5}(1-\beta)^{-3 / 5}\right)\left[2^{-2 / 5}\left(\frac{\beta}{1-\beta}\right)^{3 / 5}+1\right]^{-1 / 3}<0 .
\end{aligned}
$$

Moreover we have that

$$
g(1 / 3) \approx 0.036>0, \quad \text { while } \quad g\left(\frac{2^{2 / 3}}{1+2^{2 / 3}}\right) \approx-0.094<0,
$$

hence we define $\beta_{3}=\sup \left\{\beta \in\left(\frac{1}{3}, \frac{2^{2 / 3}}{1+2^{2 / 3}}\right) \mid g(\beta)>0\right\}$ and notice that the set

$$
\left\{\beta \in\left(\frac{1}{3}, \frac{2^{2 / 3}}{1+2^{2 / 3}}\right) \mid(3.6) \text { holds }\right\}=\left(1 / 3, \beta_{3}\right)
$$

is a nonempty interval. 
At last, we consider also the case when $\beta \in\left[\frac{2^{2 / n}}{1+2^{2 / n}}, 1\right)$. As we pointed out above, here we obtain the minimum in the right hand side of (3.5) for $m=1 / 2$. Comparing again with the functional for the ball, in the two dimensional case, we have connectedness when $2 / 3 \leq \beta<\beta_{2}$, since we have

$$
\lambda_{2}(B)<2(2-\beta) \lambda_{1}(B) .
$$

On the other hand, unfortunately, we do not obtain additional informations when $n=3$.

Putting all the above information together, we conclude connectedness for:

$n=2: \quad \beta \in\left[0, \beta_{2}\right) \approx[0,0.730)$,

$n=3: \quad \beta \in\left[0, \beta_{3}\right) \approx[0,0.476)$.

The case $0<\beta<\beta_{n}(1-\alpha)$. Recall from the above (case $\alpha=0$ ) that

$$
\eta \lambda_{2}(\Omega)+(1-\eta) \lambda_{3}(\Omega)>\eta \lambda_{2}(B)+(1-\eta) \lambda_{3}(B),
$$

for $\eta \in\left[0, \beta_{n}\right)$. This implies, with the choice $\eta=\frac{\beta}{1-\alpha}$,

$$
\frac{\beta}{1-\alpha} \lambda_{2}(\Omega)+\frac{(1-\alpha-\beta)}{1-\alpha} \lambda_{3}(\Omega)>\frac{\beta}{1-\alpha} \lambda_{2}(B)+\frac{(1-\alpha-\beta)}{1-\alpha} \lambda_{3}(B),
$$

for $\frac{\beta}{1-\alpha} \in\left[0, \beta_{n}\right)$, and so

$$
\beta \lambda_{2}(\Omega)+(1-\alpha-\beta) \lambda_{3}(\Omega)>\beta \lambda_{2}(B)+(1-\alpha-\beta) \lambda_{3}(B),
$$

for $\beta \in\left[0, \beta_{n}(1-\alpha)\right)$. Together with $\lambda_{1}(\Omega) \geq \lambda_{1}(B)$ this concludes the proof of Theorem 1.1 (iii).

Proof of Theorem 1.1 (iv): the case $\beta=0$. We finally consider the case $\beta=0$, that is, we deal with the functional $\alpha \lambda_{1}(\cdot)+(1-\alpha) \lambda_{3}(\cdot)$. To prove connectedness we first look for the best disconnected set.

Claim C. Let $\beta=0$. A disconnected minimiser is made by a ball supporting $u_{3}$ and another set supporting $u_{1}, u_{2}$.

Proof of Claim C. First of all we note that a configuration with a ball supporting the first eigenfunction and a set supporting the others would be three balls using the Krahn-Szegö inequality (1.5) on this last set. A set made by three balls is as required in the claim.

On the other hand an optimal configuration with a ball supporting the second eigenvalue would have $\lambda_{2}(\Omega)=$ $\lambda_{3}(\Omega)$, as scaling down the ball to obtain this does not effect $\lambda_{1}(\Omega), \lambda_{3}(\Omega)$. Up to switch $\lambda_{2}$ and $\lambda_{3}$ we are in a configuration with a ball supporting $u_{3}$ and another set supporting $u_{1}, u_{2}$. So the claim is proved.

We can now focus on a disconnected set made by a ball with measure $m$ supporting $u_{3}$ and a set (with measure $1-m$ ) supporting $u_{1}, u_{2}$; we aim to rule it out. This is done by obtaining lower bounds for the first and third eigenvalues and using comparison with a ball. By the scaling (1.3), we have $\lambda_{3}(\Omega)=\frac{\lambda_{1}(B)}{m^{2 / n}}$, while the Faber-Krahn and the Krahn-Szegö inequalities respectively give

$$
\lambda_{1}(\Omega) \geq \frac{\lambda_{1}(B)}{(1-m)^{2 / n}} \quad \text { and } \quad \frac{\lambda_{1}(B)}{m^{2 / n}}=\lambda_{3}(\Omega) \geq \lambda_{2}(\Omega) \geq 2^{2 / n} \frac{\lambda_{1}(B)}{(1-m)^{2 / n}},
$$

which implies $\frac{1}{m} \geq 3$, and so $m \leq \frac{1}{3}$. By [12], Corollary 5.2.2 we have that, since $\Omega$ is disconnected, for $n=2,3$

$$
\lambda_{3}(B)=\lambda_{2}(B) \leq \lambda_{3}(\Omega) .
$$

By the Faber-Krahn inequality, the ball strictly lowers the first eigenvalue, so we rule out this configuration for all $\alpha \in[0,1]$ when $n=2,3$. 
For $n \geq 4$ we must be more precise and obtain only partial estimates. If $\lambda_{3}(\Omega)=\frac{\lambda_{1}(B)}{m^{2 / n}} \geq \frac{\lambda_{2}(B)}{(1-m)^{2 / n}}$, then as we assume $\beta=0$, the set supporting the first two eigenvalues should be a ball $B_{1}$. This would contradict the optimality of $\Omega$, as we would have $\lambda_{3}\left(B_{1}\right)=\lambda_{2}\left(B_{1}\right) \leq \lambda_{3}(\Omega)$ and $\left|B_{1}\right|<1$. So we conclude $\frac{\lambda_{1}(B)}{m^{2 / n}}<\frac{\lambda_{2}(B)}{(1-m)^{2 / n}}$, hence $m>\frac{m_{0}^{n / 2}}{1+m_{0}^{n / 2}}$, which gives the bound

$$
\frac{1}{1-m}>1+m_{0}^{n / 2}
$$

Taking into account (3.7), (3.8) and the estimate $m \geq 1 / 3$, we obtain

$$
\alpha \lambda_{1}(\Omega)+(1-\alpha) \lambda_{3}(\Omega) \geq \alpha \lambda_{1}(B)\left(1+m_{0}^{n / 2}\right)^{2 / n}+(1-\alpha) \lambda_{1}(B) 3^{2 / n} .
$$

By comparing the lower bound (3.9) with $\alpha \lambda_{1}(B)+(1-\alpha) \lambda_{3}(B)$ we deduce that a minimiser is connected when $\gamma_{n}<\alpha \leq 1$ and the Proof of Theorem 1.1 (iv) is concluded. We have connectedness, for example, in the following ranges:

$$
\begin{array}{ll}
n=2: & \alpha \in[0,1] ; \\
n=3: & \alpha \in[0,1] ; \\
n=4: & \alpha \in\left(\gamma_{4}, 1\right] \approx(0.311,1] ; \\
n=5: & \alpha \in\left(\gamma_{5}, 1\right] \approx(0.467,1] ; \\
n=6: & \alpha \in\left(\gamma_{6}, 1\right] \approx(0.547,1] .
\end{array}
$$

\section{The TWO DIMENSIONAL CASE}

We start this section with a lemma which rules out a minimiser for problem (1.2) with three connected components when $n=2$. We remind that we call $\mathcal{F}(\cdot)=\alpha \lambda_{1}(\cdot)+\beta \lambda_{2}(\cdot)+(1-\alpha-\beta) \lambda_{3}(\cdot)$, while $\mathcal{G}$ is the same functional for $\alpha=0$, in order to avoid confusion.

\section{Lemma 4.1. Let $n=2$. Any disconnected minimiser of (1.2) has at most two connected components.}

Proof. For the case $\alpha+\beta=1$, which corresponds to the functional $\alpha \lambda_{1}(\cdot)+(1-\alpha) \lambda_{2}(\cdot)$, it is clear that a minimiser has at most two components since only the first two eigenvalues are into play. For $\alpha+\beta<1$ the Faber-Krahn inequality implies that a disconnected minimiser with three components would be the union of three disjoint balls. If $\alpha>0$, it is possible to apply Lemma 2.3 to the union of the balls supporting the second and the third eigenfunctions, thus ruling out this configuration. For $\alpha=0$ (that is, for the functional $\left.\beta \lambda_{2}(\cdot)+(1-\beta) \lambda_{3}(\cdot)\right)$ this argument does not work, since we can lower only $\lambda_{1}$ which is not into play, while neither $\lambda_{2}$ nor $\lambda_{3}$ are lowered. Hence we rule out the configuration with three connected components only for $n=2$, by comparing it with $B$ and $\Theta$.

Let $\mathcal{G}(\cdot)=\beta \lambda_{2}(\cdot)+(1-\beta) \lambda_{3}(\cdot)$, and write $\Omega_{i}, i=1,2,3$, for the three components of $\Omega$. Assuming $\lambda_{i}(\Omega)=$ $\lambda_{1}\left(\Omega_{i}\right)$ for $i=1,2,3$ gives $\left|\Omega_{1}\right| \geq\left|\Omega_{2}\right| \geq\left|\Omega_{3}\right|$. We write $m=\left|\Omega_{1}\right|$ and note that $\left|\Omega_{2}\right|=m$, as for $\left|\Omega_{1}\right|>\left|\Omega_{2}\right|$ we could enlarge $\Omega_{2}$ and shrink $\Omega_{1}$, lowering the functional. Thus $\left|\Omega_{3}\right|=1-2 m$, and the following constraints on $m$ hold:

1) Remark 2.4 implies $\lambda_{2}(B)>\lambda_{2}(\Omega)=\lambda_{1}\left(\Omega_{2}\right)=\frac{\lambda_{1}(B)}{m}$, so $m>\frac{\lambda_{1}(B)}{\lambda_{2}(B)}=m_{1} \approx 0.394$.

2) We must have $\frac{\lambda_{2}(B)}{m}=\lambda_{2}\left(\Omega_{1}\right) \geq \lambda_{1}\left(\Omega_{3}\right)=\frac{\lambda_{1}(B)}{1-2 m}$, as otherwise we can reduce to only two components. This inequality implies

$$
m \leq \frac{\lambda_{2}(B)}{\lambda_{1}(B)+2 \lambda_{2}(B)}=m_{2} \approx 0.418 .
$$


Coming back to the study of $\mathcal{G}$, we can use the scaling properties of eigenvalues and the bounds above to obtain

$$
\mathcal{G}(\Omega)=\beta \lambda_{2}(\Omega)+(1-\beta) \lambda_{3}(\Omega)=\left\{\frac{\beta}{m}+\frac{(1-\beta)}{(1-2 m)}\right\} \lambda_{1}(B) \geq\left\{\frac{\beta}{m_{2}}+\frac{(1-\beta)}{\left(1-2 m_{1}\right)}\right\} \lambda_{1}(B) .
$$

Now we look for those $\beta$ for which the unit ball $B$ gives a lower value of $\mathcal{G}$ than this lower bound. In particular we are looking for those $\beta$ that satisfy

$$
\mathcal{G}(B)-\mathcal{G}(\Omega) \leq \lambda_{2}(B)-\left\{\frac{\beta}{m_{2}}+\frac{(1-\beta)}{\left(1-2 m_{1}\right)}\right\} \lambda_{1}(B)<0
$$

i.e.

$$
\beta<\frac{\frac{1}{\left(1-2 m_{1}\right)}-\frac{1}{m_{1}}}{\left\{\frac{1}{\left(1-2 m_{1}\right)}-\frac{1}{m_{2}}\right\}} \approx 0.936
$$

For this range of $\beta$ three balls can not be optimal when minimising $\mathcal{G}$.

The remaining $\beta$ are ruled out by comparing $\Omega$ with $\Theta$. Using (4.1), three connected components can not be optimal when

$$
\mathcal{G}(\Theta)-\mathcal{G}(\Omega) \leq 2 \beta \lambda_{1}(B)+2(1-\beta) \lambda_{2}(B)-\left\{\frac{\beta}{m_{2}}+\frac{(1-\beta)}{\left(1-2 m_{1}\right)}\right\} \lambda_{1}(B)<0
$$

i.e. when

$$
\beta>\frac{2 \lambda_{2}(B)-\frac{\lambda_{1}(B)}{1-2 m_{1}}}{2 \lambda_{2}(B)-\left(2+\frac{1}{1-2 m_{1}}-\frac{1}{m_{2}}\right) \lambda_{1}(B)} \approx 0.479 .
$$

Since the two ranges we obtained on $\beta$ cover all cases, a minimiser for (1.2) can never have three components in $\mathbb{R}^{2}$.

We prove now an important lemma, which asserts that a disconnected minimiser must have multiple eigenvalues. The idea of the proof is that if every eigenvalue is simple, then small variations of the connected components (in the sense of shrinking one and enlarging the other) contradict the optimality of such a disconnected set. For simplicity we will often write $\lambda_{i}=\lambda_{i}(\Omega), \gamma=1-\alpha-\beta$, and as before define $m_{0}=\frac{\lambda_{1}(B)}{\lambda_{2}(B)} \approx 0.394$.

Lemma 4.2. A disconnected minimiser $\Omega$ for $(1.2)$ in $\mathbb{R}^{2}$ can not have both $\lambda_{1}(\Omega) \neq \lambda_{2}(\Omega)$ and $\lambda_{2}(\Omega) \neq \lambda_{3}(\Omega)$.

Proof. Note that we only need to consider the cases for problem (1.2) that are not covered by Remark 1.2. Additionally, the case of three components is ruled out by Lemma 4.1. The analysis of the remaining cases is divided into three steps.

Step I. We consider the case of a set $\Omega=\Omega_{1} \cup \Omega_{2}$, with $\Omega_{1}$ supporting $u_{1}$, while $\Omega_{2}$ supports $u_{2}$ and $u_{3}$. From the hypotheses of the Step, $\lambda_{1}=\lambda_{1}\left(\Omega_{1}\right), \lambda_{2}=\lambda_{1}\left(\Omega_{2}\right)$ and $\lambda_{3}=\lambda_{2}\left(\Omega_{2}\right)$, and by Faber - Krahn $\Omega_{1}$ is a ball. We define $m=\left|\Omega_{1}\right|$, so $1-m=\left|\Omega_{2}\right|$. The following constraints on $m$ hold:

1) $m>\frac{\lambda_{1}(B)}{\lambda_{2}(B)}=m_{1} \approx 0.394$, since $\frac{\lambda_{1}(B)}{m}=\lambda_{1}\left(\Omega_{1}\right) \leq \lambda_{1}\left(\Omega_{2}\right)=\lambda_{2}<\lambda_{2}(B)$ (see Rem. 2.4).

2) $\frac{\lambda_{2}(B)}{m}=\lambda_{2}\left(\Omega_{1}\right) \geq \lambda_{2}\left(\Omega_{2}\right)>\frac{\lambda_{2}(\Theta)}{(1-m)}=\frac{2 \lambda_{1}(B)}{(1-m)}$, so $m<\frac{\lambda_{2}(B)}{2 \lambda_{1}(B)+\lambda_{2}(B)}=m_{2} \approx 0.559$.

Now we can shrink $\Omega_{1}$ and enlarge $\Omega_{2}$, in order to obtain two new sets of the same shape $\widetilde{\Omega}_{1}, \widetilde{\Omega}_{2}$, such that $\left|\widetilde{\Omega}_{1}\right|=m-\varepsilon$, while $\left|\widetilde{\Omega}_{2}\right|=1-m+\varepsilon$. Writing $\widetilde{\lambda}_{i}$ for the eigenvalues of $\widetilde{\Omega}_{1} \cup \widetilde{\Omega}_{2}$ we obtain the following ratios (for $\varepsilon \ll 1)$ :

$$
\frac{\widetilde{\lambda}_{1}}{\lambda_{1}}=\frac{m}{m-\varepsilon} \approx 1+\frac{\varepsilon}{m} ; \quad \frac{\tilde{\lambda}_{2}}{\lambda_{2}}=\frac{\widetilde{\lambda}_{3}}{\lambda_{3}}=\frac{(1-m)}{1-m+\varepsilon} \approx 1-\frac{\varepsilon}{1-m} .
$$


The optimality of $\Omega$ implies $\mathcal{F}(\Omega) \leq \mathcal{F}\left(\widetilde{\Omega}_{1} \cup \widetilde{\Omega}_{2}\right)$, that means

$$
\left(\frac{\alpha \lambda_{1}}{m}-\frac{\beta \lambda_{2}+\gamma \lambda_{3}}{1-m}\right) \varepsilon+o(\varepsilon) \geq 0 .
$$

Taking either $\varepsilon>0$ or $\varepsilon<0$ (this is possible since we are supposing that the eigenvalues are simple) gives that the expression in the brackets must be zero, hence $\frac{\alpha \lambda_{1}}{m}=\frac{\beta \lambda_{2}+\gamma \lambda_{3}}{1-m}$.

In order to conclude this first step it suffices to consider $\varepsilon>0$. Since $\widetilde{\lambda}_{3} \leq \lambda_{3}$, we have a contradiction if $\mathcal{F}\left(\widetilde{\Omega}_{1} \cup \widetilde{\Omega}_{2}\right)<\mathcal{F}(\Omega)$, i.e. when

$$
\alpha \widetilde{\lambda}_{1}+\beta \widetilde{\lambda}_{2}<\alpha \lambda_{1}+\beta \lambda_{2}
$$

Equation (4.2) holds if and only if

$$
\beta \lambda_{2}\left(1-\frac{\widetilde{\lambda}_{2}}{\lambda_{2}}\right)>\alpha \lambda_{1}\left(\frac{\widetilde{\lambda}_{1}}{\lambda_{1}}-1\right) \quad \Longleftrightarrow \quad \beta>\alpha\left(\frac{\lambda_{1}}{\lambda_{2}}\right)\left(\frac{1-m}{m}\right) .
$$

Using $\frac{\lambda_{1}}{\lambda_{2}} \leq \frac{\lambda_{1}(B)}{m} \frac{1-m}{\lambda_{2}(\Theta)}=\frac{1-m}{2 m}$ and the above constraints on $m$ gives $\frac{1-m}{m} \leq \frac{1-m_{1}}{m_{1}}$. So if $\beta>\frac{\alpha}{2}\left(\frac{1-m_{1}}{m_{1}}\right)^{2} \approx$ $1.18 \alpha$, the set $\Omega=\Omega_{1} \cup \Omega_{2}$ can not be optimal. The case $\beta \leq 1.18 \alpha$ was treated in Remark 1.2, and so this concludes Step I.

Step II. We now consider the case of a set $\Omega=\Omega_{1} \cup \Omega_{2}$, with $\Omega_{1}$ supporting $u_{1}$ and $u_{3}$, while $\Omega_{2}$ supports $u_{2}$. Clearly $\lambda_{1}=\lambda_{1}\left(\Omega_{1}\right), \lambda_{2}=\lambda_{1}\left(\Omega_{2}\right)$ and $\lambda_{3}=\lambda_{2}\left(\Omega_{1}\right)$, and again it is better to take $\Omega_{2}$ to be a ball. Write $m=\left|\Omega_{1}\right|$ and $1-m=\left|\Omega_{2}\right|$. The following constraints on $m$ hold:

1) $\frac{\lambda_{1}(B)}{m}<\lambda_{1}\left(\Omega_{1}\right) \leq \lambda_{1}\left(\Omega_{2}\right)=\frac{\lambda_{1}(B)}{(1-m)}$, so $m>1 / 2=m_{1}$.

2) $\frac{\lambda_{1}(B)}{(1-m)}=\lambda_{2}(\Omega)<\lambda_{2}(B)$ by Remark 2.4, so $m<\frac{\lambda_{2}(B)-\lambda_{1}(B)}{\lambda_{2}(B)}=1-m_{0}=m_{2} \approx 0.606$.

As in the previous case we shrink $\Omega_{1}$ to $\widetilde{\Omega}_{1}$ and we enlarge $\Omega_{2}$ to $\widetilde{\Omega}_{2}$, so that $\left|\widetilde{\Omega}_{1}\right|=m-\varepsilon$, while $\left|\widetilde{\Omega}_{2}\right|=1-m+\varepsilon$. With the same arguments of the previous Step, if $\Omega$ is optimal then $\mathcal{F}\left(\widetilde{\Omega}_{1} \cup \widetilde{\Omega}_{2}\right) \geq \mathcal{F}(\Omega)$ and so

$$
\left(\frac{\alpha \lambda_{1}+\gamma \lambda_{3}}{m}-\frac{\beta \lambda_{2}}{1-m}\right) \varepsilon+o(\varepsilon) \geq 0
$$

Taking again either $\varepsilon>0$ or $\varepsilon<0$ gives $\frac{\beta \lambda_{2}}{1-m}=\frac{\alpha \lambda_{1}+\gamma \lambda_{3}}{m}$. Now, since $\Omega_{2}$ is a ball and thanks to the bounds on $m$, we can rewrite the complete functional in a more interesting way

$$
\mathcal{F}(\Omega)=\alpha \lambda_{1}+\beta \lambda_{2}+\gamma \lambda_{3}=\frac{m}{1-m} \beta \lambda_{2}+\beta \lambda_{2}=\frac{\beta \lambda_{2}}{1-m}=\frac{\beta \lambda_{1}(B)}{(1-m)^{2}} \geq \frac{\beta \lambda_{1}(B)}{\left(1-m_{1}\right)^{2}} \geq 4 \beta \lambda_{1}(B) .
$$

Comparing this lower bound with the case of the ball gives a contradiction for $\beta$ such that

$$
\mathcal{F}(B)-\mathcal{F}(\Omega) \leq \alpha \lambda_{1}(B)+(1-\alpha) \lambda_{2}(B)-4 \beta \lambda_{1}(B)<0,
$$

i.e. for

$$
\beta>\frac{1}{4 m_{0}}-\frac{\alpha}{4}\left(\frac{1}{m_{0}}-1\right) \approx 0.635-0.385 \alpha .
$$

In order to consider the cases that are not covered by Remark 1.2, we look at the equations $\alpha=\alpha_{2}, \beta=$ $\frac{1}{4 m_{0}}-\frac{\alpha}{4}\left(\frac{1}{m_{0}}-1\right)$, and $\beta=\beta_{2}(1-\alpha)$. The remaining cases can be viewed as inside a small triangle in the $\alpha-\beta$ plane, with vertices approximately given by $A=(0.275 ; 0.529), B=(0.350 ; 0.500)$ and $C=(0.350 ; 0.474)$ (See Fig. 2). 


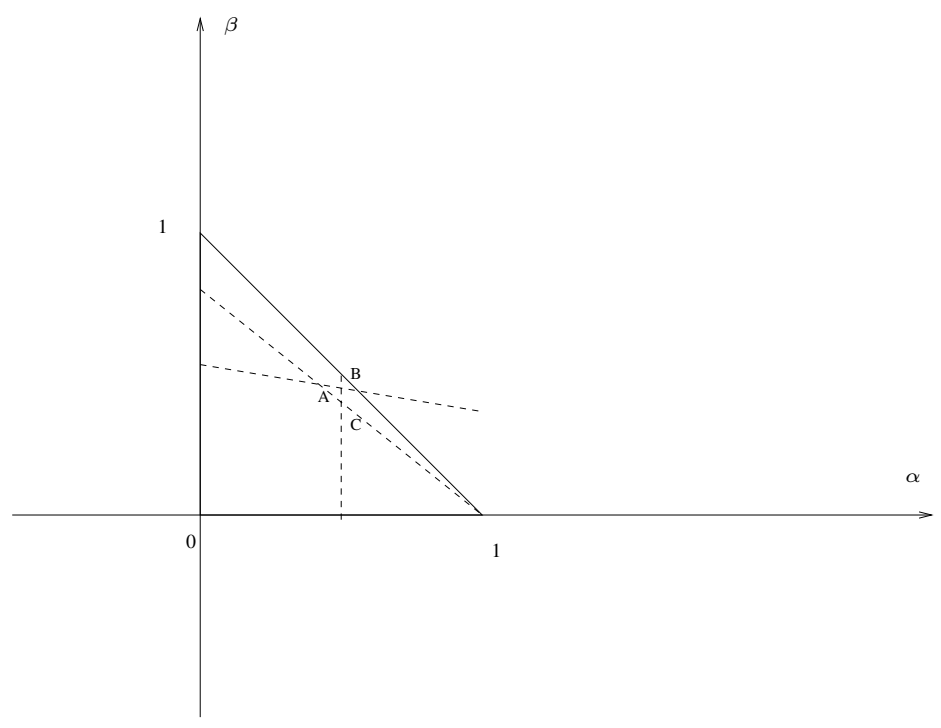

Figure 2. The small triangle in the $\alpha-\beta$ plane.

For these remaining points, it is possible to show that a ball is better than $\Omega$, i.e. that $\mathcal{F}(B)-\mathcal{F}(\Omega)<0$. In fact, the following relations hold between the eigenvalues of the ball and those of $\Omega$ (using $m \in\left(m_{1}, m_{2}\right)$, the Faber-Krahn and the Krahn-Szegö inequalities):

$$
\begin{aligned}
& \lambda_{1}(B)-\lambda_{1} \leq\left(1-\frac{1}{m}\right) \lambda_{1}(B) \leq\left(1-\frac{1}{m_{2}}\right) \lambda_{1}(B), \\
& \lambda_{2}(B)-\lambda_{2}=\lambda_{2}(B)-\frac{\lambda_{1}(B)}{1-m} \leq \lambda_{2}(B)-2 \lambda_{1}(B), \\
& \lambda_{3}(B)-\lambda_{3} \leq \lambda_{2}(B)-\frac{2 \lambda_{1}(B)}{m} \leq \lambda_{2}(B)-\frac{2 \lambda_{1}(B)}{m_{2}} .
\end{aligned}
$$

From (4.4) we get

$$
\mathcal{F}(B)-\mathcal{F}(\Omega) \leq \alpha \lambda_{1}(B)\left(1-\frac{1}{m_{2}}\right)+\beta\left(\lambda_{2}(B)-2 \lambda_{1}(B)\right)+(1-\alpha-\beta)\left(\lambda_{2}(B)-\frac{2 \lambda_{1}(B)}{m_{2}}\right) .
$$

Hence the ball is better than $\Omega$ if

$$
\beta<\frac{2 \lambda_{1}(B)-m_{2} \lambda_{2}(B)}{2 \lambda_{1}(B)-2 m_{2} \lambda_{1}(B)}+\alpha \frac{\left(-\lambda_{1}(B)+m_{2}\left(\lambda_{2}(B)-\lambda_{1}(B)\right)\right)}{2 \lambda_{1}(B)-2 m_{2} \lambda_{1}(B)} \approx 0.58-0.08 \alpha .
$$

This inequality together with (4.3) concludes Step II.

Step III. We now consider the case of a set $\Omega=\Omega_{1} \cup \Omega_{2}$, with $\Omega_{1}$ supporting $u_{1}$ and $u_{2}$, while $\Omega_{2}$ supports $u_{3}$. Clearly $\lambda_{1}=\lambda_{1}\left(\Omega_{1}\right), \lambda_{2}=\lambda_{2}\left(\Omega_{1}\right)$ and $\lambda_{3}=\lambda_{1}\left(\Omega_{2}\right)$, and it is better to take $\Omega_{2}$ to be a ball. Let $m=\left|\Omega_{1}\right|$ and $1-m=\left|\Omega_{2}\right|$. Note that if $\lambda_{3}(\Omega)=\lambda_{1}\left(\Omega_{2}\right) \geq m \lambda_{3}\left(\Omega_{1}\right)$, then $\Omega$ can not be optimal. In fact in this case it is better to take the connected set obtained by enlarging $\Omega_{1}$ till unit measure, since this lowers both $\lambda_{1}$ and $\lambda_{2}$ (by monotonicity), while also the third eigenvalue is lower, by hypothesis. The following constraints on $m$ hold:

1) $\lambda_{2}(B)>\lambda_{2}(\Omega)=\lambda_{2}\left(\Omega_{1}\right) \geq \frac{2 \lambda_{1}(B)}{m}$ (see Rem. 2.4), so $m>2 m_{0}=m_{1} \approx 0.788$. 
2 ) In order to have $\Omega$ optimal, an upper bound on $m$ follows from inequality $m \lambda_{3}\left(\Omega_{1}\right)>\lambda_{1}\left(\Omega_{2}\right)$ explained above and from the fact that $\lambda_{2}(\Omega)<\lambda_{2}(B)$ (see Rem. 2.4). Using also (2.2) from Theorem 2.1 gives

$$
\frac{\lambda_{1}(B)}{(1-m)}=\lambda_{1}\left(\Omega_{2}\right)<m \lambda_{3}\left(\Omega_{1}\right) \leq m \frac{\lambda_{2}(B)}{\lambda_{1}(B)} \lambda_{2}\left(\Omega_{1}\right)<m \frac{\lambda_{2}(B)^{2}}{\lambda_{1}(B)}
$$

This means $m^{2}-m+m_{0}^{2}<0$, which gives the upper bound

$$
m<\frac{\lambda_{2}(B)+\sqrt{\lambda_{2}(B)^{2}-4 \lambda_{1}(B)^{2}}}{2 \lambda_{2}(B)}=m_{2} \approx 0.808 .
$$

As in the previous steps we can enlarge $\Omega_{1}$ to $\widetilde{\Omega}_{1}$ and we can shrink $\Omega_{2}$ to $\widetilde{\Omega}_{2}$, in order that $\left|\widetilde{\Omega}_{1}\right|=m+\varepsilon$, while $\left|\widetilde{\Omega}_{2}\right|=1-m-\varepsilon$. The following ratios between the eigenvalues of $\widetilde{\Omega}_{1} \cup \widetilde{\Omega}_{2}$ and those of $\Omega$ hold (for $\varepsilon \ll 1$ ):

$$
\frac{\widetilde{\lambda}_{1}}{\lambda_{1}}=\frac{\widetilde{\lambda}_{2}}{\lambda_{2}}=\frac{m}{m+\varepsilon} \approx 1-\frac{\varepsilon}{m} ; \quad \frac{\widetilde{\lambda}_{3}}{\lambda_{3}}=\frac{1-m}{1-m-\varepsilon} \approx 1+\frac{\varepsilon}{1-m} .
$$

In order to be optimal, $\Omega$ must satisfy

$$
\alpha \widetilde{\lambda}_{1}+\beta \widetilde{\lambda}_{2}+(1-\alpha-\beta) \widetilde{\lambda}_{3} \geq \alpha \lambda_{1}+\beta \lambda_{2}+(1-\alpha-\beta) \lambda_{3} .
$$

An analogous argument to that in Step I and Step II gives a contradiction for $\beta \gtrsim 0.914-0.948 \alpha$. Actually we can obtain a better result observing that $\Omega$ is worse than $\Omega_{1}$ enlarged to unit measure (which we will call $\bar{\Omega}$ in the following) if $\beta$ is suitably large. We denote by $\left\{\bar{\lambda}_{i}\right\}$ the eigenvalues of $\bar{\Omega}$ and we again write $\gamma=1-\alpha-\beta$ for the sake of simplicity. The following relations between the eigenvalues hold: $\bar{\lambda}_{1}=m \lambda_{1}, \bar{\lambda}_{2}=m \lambda_{2}$ and $\bar{\lambda}_{3}=m \lambda_{3}\left(\Omega_{1}\right) \leq \frac{m}{m_{0}} \lambda_{2}$, using (2.2) from Theorem 2.1 by Ashbaugh and Benguria. This gives

$$
\mathcal{F}(\bar{\Omega})=\alpha \bar{\lambda}_{1}+\beta \bar{\lambda}_{2}+\gamma \bar{\lambda}_{3} \leq \mathcal{F}(\Omega)+\alpha(m-1) \lambda_{1}+\beta(m-1) \lambda_{2}+\gamma\left(\frac{m}{m_{1}}-1\right) \lambda_{2} .
$$

Clearly $\Omega$ can not be optimal when $\mathcal{F}(\bar{\Omega})-\mathcal{F}(\Omega)<0$, which holds if

$$
\alpha(m-1) \lambda_{1}+\left[\beta(m-1)+\gamma\left(\frac{m}{m_{0}}-1\right)\right] \lambda_{2}<0 .
$$

The first part (2.1) of Theorem 2.1 gives that the result follows if

$$
\left[\alpha(m-1) m_{0}+\beta(m-1)+\gamma\left(\frac{m}{m_{0}}-1\right)\right] \lambda_{2}<0 .
$$

Since $m \in\left(m_{1}, m_{2}\right)$ and the function in brackets is clearly increasing in $m, \Omega$ can not be optimal when

$$
\frac{m_{2}}{m_{0}}-1+\alpha\left(\left(m_{2}-1\right) m_{0}+1-\frac{m_{2}}{m_{0}}\right)+\beta\left(m_{2}-\frac{m_{2}}{m_{0}}\right)<0
$$

i.e. for

$$
\beta>\frac{\left(\frac{m_{2}}{m_{0}}-1\right)+\alpha\left(\left(m_{2}-1\right) m_{0}+1-\frac{m_{2}}{m_{0}}\right)}{\frac{m_{2}}{m_{0}}-m_{2}} \approx 0.845-0.906 \alpha .
$$

In conclusion we have an estimate that tells us that when $\beta$ is suitably big, then $\Omega=\Omega_{1} \cup \Omega_{2}$ can not be optimal. Writing $\gamma=1-\alpha-\beta$, we now finally show that $\Omega$ can not be optimal also when $\gamma$ is not very small. We use a technique very similar to the case $\beta$ big. For this suppose $\Omega$ is optimal for the problem (1.2) and let 
$|\varepsilon| \ll 1$. Then if we enlarge $\Omega_{1}$ to $\widetilde{\Omega}_{1}$ with measure $m+\varepsilon$ and we shrink $\Omega_{2}$ to $\widetilde{\Omega}_{2}$ with measure $1-m-\varepsilon$, calling $\lambda_{i}$ the eigenvalues of $\Omega=\Omega_{1} \cup \Omega_{2}$, while $\widetilde{\lambda}_{i}$ are the eigenvalues of $\widetilde{\Omega}=\widetilde{\Omega}_{1} \cup \widetilde{\Omega}_{2}$, we must have $\mathcal{F}(\Omega)-\mathcal{F}(\widetilde{\Omega}) \leq 0$. On the other hand, with analogous computations to those in Step II,

$$
\mathcal{F}(\Omega)-\mathcal{F}(\widetilde{\Omega})=\left(\frac{\gamma \lambda_{3}}{1-m}-\frac{\alpha \lambda_{1}+\beta \lambda_{2}}{m}\right) \varepsilon+o(\varepsilon),
$$

and hence the expression in brackets must be zero, as otherwise taking $\varepsilon>0$ or $\varepsilon<0$ (this is possible since we are treating only the case of simple eigenvalues) contradicts the optimality of $\Omega$. So if $\Omega$ is optimal then $\alpha \lambda_{1}+\beta \lambda_{2}=\gamma \lambda_{3} \frac{m}{1-m}$. Since $m \mapsto \frac{1}{(1-m)^{2}}$ is increasing we have the lower bound

$$
\mathcal{F}(\Omega, \alpha, \beta)=\gamma \lambda_{3} \frac{m}{1-m}+\gamma \lambda_{3} \geq \gamma \lambda_{1}(B) \frac{1}{\left(1-2 m_{1}\right)^{2}}
$$

We can show that, for $\gamma$ suitably big, comparing the functional for $\Theta$ with the lower bound above gives an absurd. In fact, the functional for the two balls is given by

$$
\mathcal{F}(\Theta, \alpha, \beta)=(\alpha+\beta) 2 \lambda_{1}(B)+\gamma 2 \lambda_{2}(B)=2 \lambda_{1}(B)+\gamma\left(2 \lambda_{2}(B)-2 \lambda_{1}(B)\right) .
$$

Hence $\mathcal{F}(\Theta, \alpha, \beta)<\mathcal{F}(\Omega, \alpha, \beta)$ for $\gamma>\bar{\gamma} \approx 0.104$, in which case two balls are better than our set $\Omega$. Combining the cases in which either $\gamma>\bar{\gamma}$ or (4.5) holds concludes Step III and hence the proof of the lemma.

\subsection{Proof of Theorem 1.3 (a)}

Proof of Theorem 1.3 (a). It is proved in Lemma 4.2 that any disconnected minimiser $\Omega$ has multiple eigenvalues. By Remark 1.2 every minimiser for $\inf \left\{\alpha \lambda_{1}(\Omega)+(1-\alpha) \lambda_{3}(\Omega) \mid \Omega\right.$ open in $\left.\mathbb{R}^{2},|\Omega| \leq 1\right\}$ is connected for all $\alpha \in[0,1]$, and we call $\tilde{\Omega}$ such a connected minimiser. The case $\lambda_{2}(\Omega)=\lambda_{3}(\Omega)$ is then ruled out, as it would give

$$
\begin{aligned}
\alpha \lambda_{1}(\Omega)+\beta \lambda_{2}(\Omega)+(1-\alpha-\beta) \lambda_{3}(\Omega) & =\alpha \lambda_{1}(\Omega)+(1-\alpha) \lambda_{3}(\Omega)>\alpha \lambda_{1}(\tilde{\Omega})+(1-\alpha) \lambda_{3}(\tilde{\Omega}), \\
& \geq \alpha \lambda_{1}(\tilde{\Omega})+\beta \lambda_{2}(\tilde{\Omega})+(1-\alpha-\beta) \lambda_{3}(\tilde{\Omega}) .
\end{aligned}
$$

Therefore any disconnected minimiser must satisfy $\lambda_{1}(\Omega)=\lambda_{2}(\Omega)$ and can be viewed as the union of a disk supporting the first eigenvalue with a connected set supporting the second and third, since a minimiser with three connected components was ruled out by Lemma 4.1.

\subsection{Proof of Theorem 1.3 (b)}

Proof of Theorem 1.3 (b). Let $\alpha+\beta<1$, and let $\tilde{\Omega}$ be a connected minimiser for $\inf \left\{(\alpha+\beta) \lambda_{2}(\Omega)+(1-\alpha-\right.$ $\beta) \lambda_{3}(\Omega) \mid \Omega$ open in $\left.\mathbb{R}^{2},|\Omega| \leq 1\right\}$, while there are no disconnected minimisers by hypotesis. Theorem 1.3 (a) then gives $\lambda_{1}(\Omega)=\lambda_{2}(\Omega)$ for a disconnected minimiser $\Omega$ for problem (1.2), whereby

$$
\begin{aligned}
\alpha \lambda_{1}(\Omega)+\beta \lambda_{2}(\Omega)+ & (1-\alpha-\beta) \lambda_{3}(\Omega)=(\alpha+\beta) \lambda_{2}(\Omega)+(1-\alpha-\beta) \lambda_{3}(\Omega) \\
& >(\alpha+\beta) \lambda_{2}(\tilde{\Omega})+(1-\alpha-\beta) \lambda_{3}(\tilde{\Omega}) \\
& \geq \alpha \lambda_{1}(\tilde{\Omega})+\beta \lambda_{2}(\tilde{\Omega})+(1-\alpha-\beta) \lambda_{3}(\tilde{\Omega})
\end{aligned}
$$

which contradicts the minimality of $\Omega$ and thus the proof is concluded. 


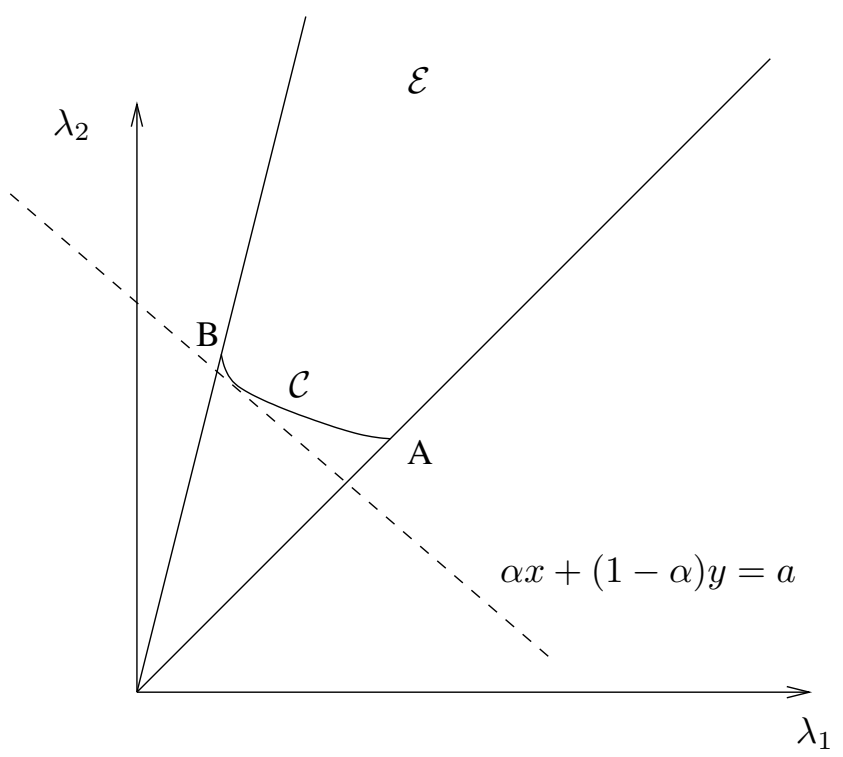

Figure 3 . The set $\mathcal{E}$.

\section{ApPENDix}

This appendix is devoted to a different proof of the connectedness of the minimisers for the problem

$$
\inf \left\{\alpha \lambda_{1}(\Omega)+(1-\alpha) \lambda_{2}(\Omega) \mid \Omega \subseteq \mathbb{R}^{2} \text {, open, with }|\Omega| \leq 1\right\}
$$

for $\alpha>0$. This corresponds to Remark 1.2 (i). It was proved in Section 3 that for problem (5.1) the unit ball is better than every disconnected set if $\alpha \in\left(\alpha_{2}, 1\right]$, while when $\alpha \in(0,1 / 2)$ the best disconnected set is the disjoint union of two equal balls, say $\Theta$. We aim to give a proof of the fact that $\Theta$ can not be optimal for (5.1) unless $\alpha=0$, that does not rely on Lemma 2.3. We focus on the case $\alpha \in(0,1 / 2)$.

We need to introduce the set

$$
\mathcal{E}=\left\{\left(\lambda_{1}(\Omega), \lambda_{2}(\Omega)\right) \mid \Omega \subseteq \mathbb{R}^{2} \text { open, with }|\Omega|=1\right\} .
$$

For a description of many properties of this set and a numerical approximation of it we refer to [8] or to ([12], Chap. 6.4). The property which interests us deals with the lower part of the boundary of $\mathcal{E}$, the curve $\mathcal{C}$ that joins the point $A=\left(\lambda_{1}(\Theta), \lambda_{2}(\Theta)\right)$ and $B=\left(\lambda_{1}(B), \lambda_{2}(B)\right)$ (see Fig. 3).

Wolf and Keller [16] proved that the curve $\mathcal{C}$ must be vertical at the point $B$ by a perturbation argument with nearly circular domains. They also suggested that $\mathcal{C}$ should be horizontal at $A$, and this was proved recently by Brasco, Nitsch and Pratelli [6]. This is the crucial point of our proof, as a minimiser for the convex combination $\alpha \lambda_{1}(\Omega)+(1-\alpha) \lambda_{2}(\Omega)$ is given by the set corresponding to the first point in which the straight line $\alpha x+(1-\alpha) y=a$ touches $\mathcal{E}$, by increasing $a$. In particular, for $\alpha=0$ this line is $y=\lambda_{2}(\Theta)=2 \lambda_{1}(B)$ by the Krahn-Szegö inequality. On the other hand, for all $\alpha \in(0,1 / 2)$, it is possible to find a set $\widetilde{\Omega}$ that is linked to a line of the form $\alpha x+(1-\alpha) y=a_{\alpha}$, with $a_{\alpha}<\lambda_{2}(\Theta)=2 \lambda_{1}(B)$, since the curve $\mathcal{C}$ has horizontal tangent. Hence $\Theta$ can not be the minimiser for (5.1) unless $\alpha=0$.

Acknowledgements. We warmly thank Michiel van den Berg and Aldo Pratelli for their suggestions on the paper. We wish to thank an anonymous referee for a very careful reading and useful suggestions. 


\section{REFERENCES}

[1] M. Abramowitz and I.A. Stegun, Handbook of Mathematical Functions with Formulas, Graphs, and Mathematical Tables. Dover Publications, New York (1972).

[2] M.S. Ashbaugh and R. Benguria, Proof of the Payne-Pölya-Weinberger conjecture. Bull. Amer. Math. Soc. 25 (1991) 19-29.

[3] M.S. Ashbaugh and R. Benguria, Isoperimetric bound for $\lambda_{3} / \lambda_{2}$ for the membrane problem. Duke Math. J. 63 (1991) 333-341.

[4] M. van den Berg, On Rayleigh's formula for the first Dirichlet eigenvalue of a radial perturbation of a ball. J. Geometric Anal. 23 (2013) 1427-1440.

[5] M. van den Berg and M. Iversen, On the minimization of Dirichlet eigenvalues of the Laplace operator. J. Geometric Anal. 23 (2013) 660-676.

[6] L. Brasco, C. Nitsch and A. Pratelli, On the boundary of the attainable set of the Dirichlet spectrum. Z. Angew. Math. Phys. 64 (2013) 591-597.

[7] D. Bucur and G. Buttazzo, Variational methods in shape optimization problems. Prog. Nonlinear Differ. Eq. Appl. Birkhäuser Verlag, Boston (2005).

[8] D. Bucur, G. Buttazzo and I. Figueiredo, On the attainable eigenvalues of the Laplace operator. SIAM J. Math. Anal. 30 (1999) 527-536.

[9] D. Bucur and A. Henrot, Minimization of the third eigenvalue of the Dirichlet Laplacian. Proc. Roy. Soc. London 456 (2000) 985-996.

[10] G. Buttazzo and G. Dal Maso, An existence result for a class of shape optimization problems. Arch. Rational Mech. Anal. 122 (1993) 183-195.

[11] R. Courant and D. Hilbert, Methods of Mathematical Physics, vol. 2. Wiley-VCH, New York (1962).

[12] A. Henrot, Extremum Problems for Eigenvalues of Elliptic Operators. Frontiers Math. Birkhäuser Verlag, Basel (2006).

[13] D. Mazzoleni and A. Pratelli, Existence of minimizers for spectral problems. J. Math. Pures Appl. 100 (2013) $433-453$. DOI: http://dx.doi.org/10.1016/j.matpur.2013.01.008.

[14] B. Osting and C.-Y. Kao, Minimal convex combinations of three sequential Laplace-Dirichlet eigenvalues, Appl. Math. Optim. 69 (2014) 123-139.

[15] S.A. Wolf, Asymptotic and Numerical Analysis of Linear and Nonlinear Eigenvalue Problems, Ph.D. Thesis. Stanford University (1993).

[16] S.A. Wolf and J.B. Keller, Range of the First Two Eigenvalues of the Laplacian. Proc. R. Soc. London A 447 (1994) $397-412$. 